\title{
Fault-Tolerant Ring Embedding in Faulty Arrangement Graphs
}

\author{
Sun-yuan Hsieh \\ Dept. of Computer Science \& Info. Engg. \\ National Taiwan University, Taiwan \\ e-mail: d3506013@csie.ntu.edu.tw
}

\author{
Gen-Huey Chen \\ Dept. of Computer Science \& Info. Engg. \\ National Taiwan University, Taiwan \\ e-mail: ghchen@csie.ntu.edu.tw
}

Chin-Wen Ho

Dept. of Computer Science \& Info. Engg.

National Central University, Taiwan

e-mail: hocw@csie.ncu.edu.tw

\begin{abstract}
The arrangement graph $A_{n, k}$, which is a generalization of the star graph $(n-k=1)$, presents more flexibility than the star graph in adjusting the major design parameters: number of nodes, degree, and diameter. Previously the arrangement graph has proven hamiltonian. In this paper we further show that the arrangement graph remains hamiltonian even if it is faulty. Let $\left|F_{e}\right|$ and $\left|F_{v}\right|$ denote the numbers of edge faults and vertex faults, respectively. We show that $A_{n, k}$ is hamiltonian when (1) ( $k=2$ and $n-k \geq 4$, or $k \geq 3$ and $\left.n-k \geq 4+\left\lceil\frac{k}{2}\right\rceil\right)$, and $\left|F_{\epsilon}\right| \leq k(n-k)-2$, or (2) $k \geq 2, n-k \geq 2+\left\lceil\frac{k}{2}\right\rceil$, and $\left|F_{e}\right| \leq k(n-k-3)-1$, or (3) $k \geq 2, n-k \geq 3$, and $\left|F_{\varepsilon}\right| \leq k$.
\end{abstract}

\section{Introduction}

The star graph [1] has been recognized as an attractive alternative to the hypercube network. Unfortunately, there is a major drawback for the star graph: a rigorous restriction to its size (i.e., $n$ !). Recently, the arrangement graph [4], which is a generaliztion of the star graph, has been proposed as a possible solution to the problem. The arrangement graph presents more flexibility than the star graph in terms of choosing the major design parameters: number of vertices, degree, and diameter, while preserving most of the nice properties of the star graph. Previous work on the arrangement graph can be found in [3] [4] [5] [6].

Since node faults and link faults may happen when a network is put in use, it is practically meaningful to consider faulty networks. Many related works have appeared in the literature, for example [7] [8] [9] [10]. In [8], Latifi, Zheng, and Bagherzadeh showed that an $n$-dimensional hypercube with at most $n-2$ link faults is hamiltonian. In [9], Rowley and Bose showed that with slight modifications a base- $d$ undirected de Bruijn graph with at most $d-1$ edge faults is hamiltonian. In [7], Fernandes, Friesen, and Kanevsky showed that a WK-recursive network od degree $d$ with at most $d-3$ link faults is hamiltonian. In [10], Tseng, Chang and Sheu showed that an $n$-dimensional star graph with at most $n-3$ edge faults is hamiltonian.
Although Day and Tripathi [4] have shown that the arrangement graph is hamiltonian, they have assumed the arrangement graph is fault-free. In the remainding sections, we consider faulty arrangement graphs, and investigate their hamiltonicity.

\section{Prelimiaries}

First we briefly review the arrangement graph, denoted by $A_{n, k}$, where $1 \leq k<n$.

Definition 1 The vertex set of $A_{n, k}$ is denoted by $\left\{p_{1} p_{2} \cdots p_{k} \mid p_{i} \in\{1,2, \ldots, n\}\right.$ for all $1 \leq i \leq k$ and $p_{i} \neq p_{j}$ for $\left.i \neq j\right\}$. Vertex adjacency is defined as follows: $p_{1} p_{2} \cdots p_{k}$ is adjacent to $q_{1} q_{2} \cdots q_{k}$ if and only if $p_{i} \neq q_{i}$ for some $1 \leq i \leq k$ and $p_{j}=q_{j}$ for all $1 \leq j \leq k$ and $j \neq i$.

Often $k$ is referred to as the dimensions of $A_{n, k}$, and the position of $p_{i}$ is said to be the $i$ th dimension. An edge of $A_{n, k}$ is said to be of the ith dimension if its two incident vertices differ between their corresponding arrangements in the $i$ th dimension.

The structure of $A_{n, k}$ is recursive. We note that $A_{n, k}$ contains embedded $A_{n-k+r, r}$ for all $1 \leq r \leq k$. An embedded $A_{n-k+r, r}$ is conveniently denoted by $\left\langle s_{1} s_{2} \ldots s_{k}\right\rangle_{n, r}$, where $s_{i} \in\{*, 1,2, \ldots, n\}$ for all $1 \leq$ $i \leq k$ (* represents a "don"t care" symbol) and exactly $r$ of $s_{1}, s_{2}, \ldots, s_{k}$ are $*$. Two basic operations on $\left\langle s_{1} s_{2} \ldots s_{k}\right\rangle_{n, r}$ are defined as follows.

Definition 2 Suppose $\left\langle s_{1} s_{2} \ldots s_{k}\right\rangle_{n, r}$ is an embedded $A_{n-k+r, r}$ of $A_{n, k}$. For each $s_{i}=*(1 \leq i \leq$ $k)$, we define the i-partition on $\left\langle s_{1} s_{2} \ldots s_{k}\right\rangle_{n, r}$ to be an operation that partitions the embedded $A_{n-k+r, r}$ into $n-k+r$ embedded $A_{n-k+r-1, r-1}$ 's, denoted by $\left\langle s_{1} s_{2} \ldots s_{i-1} q s_{i+1} \ldots s_{k}\right\rangle_{n, r-1}$, where $q$ is a missing element of $\{1,2, \ldots, n\}$ in $s_{1} s_{2} \ldots s_{k}$.

Definition 3 Suppose $\left\langle s_{1} s_{2} \ldots s_{k}\right\rangle_{n, r}$ represents an embedded $A_{n-k+r, r}$ of $A_{n, k}$. Let $I=\left(i_{1}, i_{2}, \ldots, i_{m}\right)$,

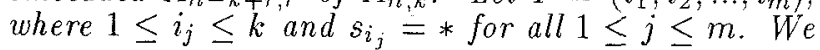


define the I-parition on $\left\langle s_{1} s_{2} \ldots s_{k}\right\rangle_{n, r}$ to be a sequence of operations that perform $i_{1}$-partition, $i_{2}$-partition,..., $i_{m}$-partition, sequentially, on $\left\langle s_{1} s_{2} \ldots s_{k}\right\rangle_{n, r}$. After the I-partition, the embedded $A_{n-k+r, r}$ is partitioned into $(n-k+r)(n-k+r-1) \cdots(n-k+r-m+1)$ embedded $A_{n-k+r-m, r-m}$ 's.

Suppose $\left\langle s_{1} s_{2} \ldots s_{k}\right\rangle_{n, r}$ and $\left\langle t_{1} t_{2} \ldots t_{k}\right\rangle_{n, r}$ represnts two embedded $A_{n-k+r, r}$ 's of $A_{n, k}$. They are said to be adjacent if there exists some $1 \leq j \leq k$ so that $s_{j} \neq *, t_{j} \neq *$, and $s_{j} \neq t_{j}$, and $\overline{s_{i}}=t_{i}$ for all $1 \leq i \leq k$ and $i \neq j$. Further, we use $\operatorname{dif}\left(\left\langle s_{1} s_{2} \ldots s_{k}\right\rangle_{n, r},\left\langle t_{1} t_{2} \ldots t_{k}\right\rangle_{n, r}\right)(=j)$ to indicate the different position between $s_{1} s_{2} \ldots s_{k}$ and $t_{1} t_{2} \ldots t_{k}$.

\section{Definition 4 Let}

$A_{0}, A_{1}, \ldots, A_{n(n-1)(n-2) \cdots(n-k+r+1)-1}$ represent embedded $A_{n-k+r, r}$ 's of $A_{n, k}$ that result from executing $a\left(i_{1}, i_{2}, \ldots, i_{k-r}\right)$-partition on $A_{n, k}$, where $i_{1} i_{2} \ldots i_{k-r}$ is a permutation of $k-r$ elements from $\{1,2, \ldots, k\}$. If $A_{i}$ is adjacent to $A_{(i-1) \bmod (n-1)(n-2) \cdots(n-k+r+1)}$ and $A_{(i+1) \bmod (n-1)(n-2) \cdots(n-k+r+1)}$ for all $0 \leq i \leq$ $n(n-1)(n-2) \cdots(n-k+r+1)-1$, then $A_{0}, A_{1}, \ldots, A_{n(n-1)(n-2) \cdots(n-k+r+1)-1}$ are said to form an r-ring $(0 \leq r \leq k)$, denoted by $R_{r}=$ $\left[A_{0}, A_{1}, \ldots, A_{n(n-1)(n-2) \cdots(n-k+r+1)-1}\right]$.

If we regard each $A_{i}$ as a supervertex called $r$. vertex, $r \geq 1$, and define a superedge called $r$-edge between $A_{i}$ and $A_{(i+1) \bmod n(n-1)(n-2) \cdots(n-k+r+1)}$, then $R_{r}$ forms a cycle of $n(n-1)(n-2) \cdots(n-k+r+1)$ $r$-vertices. Actually, each $r$-edge consists of $(n-k+$ $r-1)(n-k+r-2) \cdots(n-k)$ edges of $A_{n, k}$. Each $r$ vertex can be partitioned into $n-k+r(r-1)$-vertices, and any two of them are connected with an $(r-1)$ edge. So, each $r$-vertex can be viewed as a complete graph of $n-k+r(r-1)$-vertices. For easy reference, we use $K_{n-k+r}^{r-1}$ to denote such a complete graph in subsequent discussion.

Definition 5 We define an i-partition on $R_{r}=$ $\left[A_{0}, A_{1}, \ldots, A_{n(n-1)(n-2) \cdots(n-k+r+1)-1]}\right.$ to be a sequence of operations that perform an $i$-partition on $A_{0}$, $A_{1}, \ldots, A_{n(n-1)(n-2) \cdots(n-k+r+1)-1}$, respectively, where $1 \leq i \leq k$ and $r \geq 1$.

Lemma 2.1 For $A_{n, k}$ with $n-k \geq 3$ (resp. $n-k=$ $2)$, an $R_{r}$ of (maximal) length $n(n-1)(n-2) \cdots(n-$ $k+r+1)$ contains an $R_{r-1}$ of (maximal) length $n(n-$ 1) $(n-2) \cdots(n-k+r+1)(n-k+r)$, where $r \geq 1$ (resp. $r \geq 2)$.

Proof:

Suppose $R_{r}=\left[A_{0}, A_{1}, \ldots, A_{n(n-1)(n-2) \cdots(n-k+r+1)-1}\right]$. First a partition on $R_{r}$ is executed. Then, for $0 \leq$ $i \leq n(n-1)(n-2) \cdots(n-k+r+1)-1$, two $(r=1)$-vertices, say $X_{i}$ and $Y_{i}$, are determined in each $A_{i}$ so that $Y_{(i-1) \operatorname{modn}(n-1)(n-2) \cdots(n-k+r+1)}$ and $X_{(i+1) \operatorname{modn}(n-1)(n-2) \cdots(n-k+r+1)}$ are connected to $X_{i}$ and $Y_{i}$ through $(r-1)$-edges, respectively. The existence of $X_{i}$ and $Y_{i}$ is assured by $n-k+r \geq 4$ (because $n-k \geq 3$ and $r \geq 1$, or $n-k=2$ and $r \geq 2$ ). Since each $A_{i}$ can be viewed as a complete graph $K_{n-k+r}^{r-1}$, there exists a path, denoted by $P_{i}$, from $X_{i}$ to $Y_{i}$ that passes all $(r-1)$-vertices in $A_{i}$ exactly once. Clearly, all the $(r-1)$-vertices encountered along the path: $P_{0}$, $\left(Y_{0}, X_{1}\right), P_{1},\left(Y_{1}, X_{2}\right), \ldots, P_{n(n-1)(n-2) \cdots(n-k+r+1)-1}$, $\left(Y_{n(n-1)(n-2) \cdots(n-k+r+1)-1}, X_{0}\right)$, form an $R_{r-1}$ of the desired length.

Q.E.D.

Remark. In the rest of this paper, $X_{i}$ and $Y_{i}$ as described above are referred to as the entry $\left(r_{-1}\right)$-vertex and the exit $(r-1)$-vertex of $A_{i}$, respectively.

Given a graph $G$, we use $V(G)$ and $E(G)$ to represent the sets of vertices and edges of $G$, respectively. We say $G$ is Connected if there exists a path in $G$ between any two of its vertices. The degree of $v \in V(G)$, denoted by $\operatorname{deg}(v)$, is the number of edges incident with it. A cycle (path) in $G$ is called a hamiltonian $c y$ cle $(p a t h)$ if it contains every vertex of $G$ exactly once. $G$ is said to be hamiltonian if it contains a hamiltonian cycle. A hamiltonian graph $G$ is said to be $k$ vertex (edge) fault-tolerant hamiltonian if it remains hamiltonian after removing at most $k$ vertices (edges) [11]. $G$ is said to be hamiltonian-connected if there exists a hamiltonian path between every two vertices of $G$.

Definition 6 Suppose $G$ is a hamiltonian graph. The vertex (resp. edge) fault-tolerant hamiltonicity of $G$, denoted by $H_{v}(G)$ (resp. $H_{e}(G)$ ), is defined as $\max \{k\lfloor G$ is $k$ vertex (resp. edge) fault-tolerant hamiltonian $\}$.

Lemma $2.2[2]$ If $|V(G)| \geq 3$ and $\operatorname{deg}(u)+\operatorname{deg}(v) \geq$ $|V(G)|$ for every two non-adjacent vertices $u, v$ of $\bar{G}$, then $G$ is hamiltonian.

Lemma 2.3 [2] If $G$ is a connected graph and $\operatorname{deg}(u)+\operatorname{deg}(v) \geq|V(G)|+1$ for every two nonadjacent vertices $\bar{u}, v$ of $G$, then $G$ is hamiltonianconnected.

Lemma 2.4 Removing any $d-3$ edges $\left(x_{1}, y_{1}\right)$, $\left(x_{2}, y_{2}\right), \ldots,\left(x_{d-3}, y_{d-3}\right)$ from $K_{d}$ (the complete graph of d vertices) results in a hamiltonian graph (denoted by $\left.K_{d}^{\prime}\right)$. Further, if for each $\left(x_{i}, y_{i}\right), 1 \leq i \leq d-3$, there exists another $\left(x_{j}, y_{j}\right), j \neq i$, so that they are not incident to the same vertex, then $K_{d}^{\prime}$ is hamiltonianconnected.

Proof: We first prove that $K_{d}^{\prime}$ is hamiltonian by showing $\operatorname{deg}\left(x_{i}\right)+\operatorname{deg}\left(y_{i}\right) \geq d$ for all $1 \leq i \leq d-3$. Let $p$ and $q$ denote the number of removed edges, exclusive of $\left(x_{i}, y_{i}\right)$, that are incident to $x_{i}$ and $y_{i}$, respectively. Clearly, we have $p+q \leq d-4$, and so $\operatorname{deg}\left(x_{i}\right)+\operatorname{deg}\left(y_{i}\right)=(d-1-(p+1))+(\bar{d}-1-(q+1))=$ $2 d-4-(p+q) \geq 2 d-4-(d-4)=d$. By Lemma 2.2 , $K_{d}^{\prime}$ is hamiltonian. It can be proved similarly that $K_{d}^{\prime}$ is hamiltonian-connected if the condition is satisfied. We have $p+q \leq d-5$ under the condition, and thus $\operatorname{deg}\left(x_{i}\right)+\operatorname{deg}\left(y_{i}\right) \geq d+1$. By Lemma $2.3, K_{d}^{\prime}$ is hamiltonian-connected.

Q.E.D.

Slight modifications of the proof above can lead to the following lemma. 
Lemma 2.5 Suppose $\quad\left(x_{1}, y_{1}\right), \quad\left(x_{2}, y_{2}\right), \ldots$, $\left(x_{d-2}, y_{d-2}\right)$ are any $d-2$ edges of $K_{d}$. If for each $\left(x_{i}, y_{i}\right), 1 \leq i \leq d-2$, there exists another $\left(x_{j}, y_{j}\right)$, $j \neq i$, so that they are not incident to the same vertex, then removing $d-2$ edges from $K_{d}$ results in a hamiltonian graph.

The following two lemmas can be proved similarly.

Lemma 2.6 Removing any $d-4$ edges from $K_{d}$ results a hamiltonian-connected graph.

Lemma 2.7 Removing any $d-6$ edges and any two vertices of $K_{d}$ results in a hamiltonian-connected graph.

\section{Fault-Free Hamiltonian Cycles in $A_{n, k}$ with Faulty Edges}

Let $F_{e}$ denote the set of faulty edges. According to the dimensions where faulty edges belong to, $F_{e}$ can be partitioned into $k$ subsets, denoted by $D_{i}, i=$ $1,2, \ldots, k$, where each $D_{i}$ is the set of faulty edges that belong to the $i$ th dimension.

Lemma 3.1 Suppose $\left|F_{e}\right|=k(n-k)-2$, let $S=$ $a_{1} a_{2} \ldots a_{k}$ be a permutation of $1,2, \ldots, k$ so that $\left|D_{a_{1}}\right| \geq$ $\left|D_{a_{2}}\right| \geq \cdots \geq\left|D_{a_{k}}\right|$. Then, we have $\left|D_{a_{k}}\right| \leq n-3$ if $k=1$, and $\left|\bar{D}_{a_{k}}\right| \leq(n-k)-1$ if $k \geq 2$.

Proof: We have $\left|D_{a_{k}}\right| \leq \frac{\left|F_{\mathrm{e}}\right|}{k} \leq \frac{(k(n-k)-2)}{k}$. Thus the maximal value of $\left|D_{a_{k}}\right|$ is $\left\lfloor\frac{(k(n-k)-2)}{k}\right\rfloor$, which is equal to $n-3$ as $k=1$, and $(n-k)-1$ as $k \geq 2$. Q.E.D.

Lemma 3.2 Suppose $U=\left\langle u_{1} u_{2} \ldots u_{k}\right\rangle_{n, r}, V=$ $\left\langle v_{1} v_{2} \ldots v_{k}\right\rangle_{n, r}$, and $W=\left\langle w_{1} w_{2} \ldots w_{k}\right\rangle_{n, r}$, are arbitrary three consecutive $r$-vertices in $R_{r}$, where $r \geq 1$. Also let $p=\operatorname{dif}(U, V)$ and $q=\operatorname{dif}(V, W)$. If $\vec{u}_{p} \neq w_{q}$, then after executing a partition on $R_{r}$ each $(r-1)$ vertex of $V$ is adjacent to one $(r-1)$-vertex of $U$ or one $(r-1)$-vertex of $W$.

Proof: Without loss of generality, we assume that a $j$ partition is executed on $R_{r}$, where $1 \leq j \leq k$. Hence, we have $u_{j}=v_{j}=w_{j}=*$. Since $p \equiv \operatorname{dif}(U, V)$ and $q=\operatorname{dif}(V, W)$, we have $u_{p} \neq v_{p}, v_{q} \neq w_{q}, u_{i}=v_{i}$ for all $1 \leq i \leq k$ and $i \neq p$, and $v_{i}=w_{i}$ for all $1 \leq i \leq k$ and $\bar{i} \neq q$. Suppose $u_{p} \neq w_{q}$, and after the $j$-partition, there exists an $(r-1)$-vertex, say $V^{\prime}=$ $\left\langle v_{1} v_{2} \ldots v_{j-1} v^{\prime} v_{j+1} \ldots v_{k}\right\rangle_{n, r-1}$, of $V$ that is not adjacent to any $(r-1)$-vertex of $U$ and $W$. Thus, we have $v^{\prime}=$ $u_{p}$ for otherwise $V^{\prime}$ is adjacent to some $(r-1)$-vertex of $U$. Similarly, we have $v^{\prime}=w_{q}$. This implies $u_{q}=$ $v^{\prime}=w_{q}$, which contradicts our assumption. Q.E.D.

For $1 \leq i \leq k-1$, an $i$-edge of $A_{n, k}$ is faulty if it contains faulty edges. An $R_{r}$ of $A_{n, k}$ is said to be fault-free if it does not contain faulty $r$-edges. In the rest of this section, we use $E_{i, j}$ to denote the set of faulty $i$-edges that belong to the $j$ th dimension.

Lemma 3.3 Suppose $\left|F_{e}\right|=k(n-k)-2$, and let $S=a_{1} a_{2} \ldots a_{k}$ be a permutation of $1,2, \ldots, k$ so that $\left|D_{a_{1}}\right| \geq\left|D_{a_{2}}\right| \geq \cdots \geq\left|D_{a_{k}}\right|$. If $\left|D_{a_{1}}\right|=\left|D_{a_{2}}\right|=\cdots=$ $\left|D_{a_{k-2}}\right|=n-\bar{k}$, there is a fault-free $R_{2}$ in $A_{n, k}$.
Proof: First we construct fault-free $R_{k-1}, R_{k-2}, \ldots, R_{3}$ for the faulty $A_{n, k}$. We only need to apply a $\left(a_{1}, a_{2}, \ldots, a_{k-3}\right)$ - partition to the faulty $A_{n, k}$. Initially, an $a_{1}$-partition is executed on the faulty $A_{n, k}$, and so a $K_{n}^{k-1}$ is obtained. We note that all edges of the $K_{n}^{k-1}$ are $(k-1)$-edges and $\left|E_{k-1, a_{1}}\right|\left(\leq\left|D_{a_{1}}\right|=n-k\right)$ of them are faulty. By Lemma 2.4, the $K_{n}^{k-1}$ with $\left|E_{k-1, a_{1}}\right|$ faulty $(k-1)$-edges removed is hamiltonian. Hence, a fault-free $R_{k-1}$ can be generated.

In general, when an $a_{i}$-partition is executed on a fault-free $R_{k-i+1}$ for $2 \leq i \leq k-3$, each $(k-i+1)$ vertex of the fault-free $R_{k-i+1}$ forms a $K_{n-i+1}^{k-i}$. Since $\left|E_{k-i, a_{i}}\right| \leq\left|D_{a_{i}}\right|=n-k$, each $K_{n-i+1}^{k-i}$ contains $n-k$ faulty $(k-i)$-edges at most. Now that each $K_{n-i+1}^{k-i}$ contains $n-i+1 \geq n-k+4(k-i)$-vertices, Lemma 2.6 assures that it is hamiltonian-connected, even if all faulty $(k-i)$-edges are removed. As a result, there is a fault-free hamiltonian path for each $K_{n-i+1}^{k-i}$ that goes from the entry $(k-i)$-vertex to the exit $(k-i)$ vertex. All these hamiltonian paths interleaved with $(k-i)$-edges form a fault-free $R_{k-i}$

Next we show how to obtain a fault-free $R_{2}$ from a fault-free $R_{3}=\left[A_{0}, A_{1}, \ldots, A_{n(n-1)(n-2) \cdots(n-k+4)-1}\right]$. An $a_{k-2}$-partition is first executed on $R_{3}$ so that each $A_{i}$ forms a $K_{n-k+3}^{2}$, where $0 \leq i \leq n(n-1)(n-$ 2) $\cdots(n-k+4)-1$. There are totally $\left|E_{2, a_{k-2}}\right|$ $\left(\left|D_{a_{k-2}}\right|=n-k\right)$ faulty 2 -edges contained in all $A_{i}$ 's. If $\left|E_{2, a_{k-2}}\right|<n-k$ or $\left|E_{2, a_{k-2}}\right|$ faulty 2-edges are distributed over two or more $A_{i}$ 's, then it is assured by Lemma 2.6 that each $K_{n-k+3}^{2}$ with faulty 2-edges removed is hamiltonian-connected. Consequently, as described in the proof of Lemma 2.1, a fault-free $R_{2}$ can be generated by properly selecting the entry 2 vertex and the exit 2 -vertex for each $A_{i}$.

On the other hand, if $\left|E_{2, a_{k-2}}\right|=n-k$ and all faulty 2-edges are located in some $A_{t}$, then it is assured by Lemma 2.4 that the $K_{n-k+3}^{2}$ resulting from $A_{t}$ with $n-k$ faulty 2 -edges removed is hamiltonian. That is, the $K_{n-k+3}^{2}$ contains a fault-free hamiltonian cycle, denoted by $C=\left(X_{0}, X_{1}, \ldots, X_{n-k+2}, X_{0}\right)$, where each $X_{j}(0 \leq j \leq n-k+2)$ is a 2 -vertex of $A_{t}$. Since there are $n-k+22$-edges between each pair of $A_{i}$ and $A_{(i+1) \bmod n(n-1)(n-2) \cdots(n-k+4)}$, there are $n-k+22$-vertices in $C$ that are adjacent to $n-k+2$ 2-vertices of $A(t-1) \bmod n(n-1)(n-2) \cdots(n-k+4)$, and there are $n-k+22$-vertices in $C$ that are adjacent to $n-k+$ 2 2-vertices of $A_{(t+1) \operatorname{modn}(n-1)(n-2) \cdots(n-k+4)}$. Thus, there must exist a 2-edge, say $\left(X_{l}, X_{(l+1) \bmod n-k+3}\right)$, of $C$ so that $X_{l}$ and $X_{(l+1) \operatorname{modn}-k+3}$ are adja cent to 2-vertices of $A_{(t-1) \operatorname{modn}(n-1)(n-2) \cdots(n-k+4)}$ and

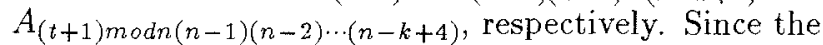
other $K_{n-k+3}^{2}$ 's that result from all $A_{j}$ 's with $j \neq t$ are hamiltonian-connceted and their 2-edges are not faulty, a fault-free $R_{2}$ can be generated by taking $X_{l}$ and $X_{(l+1) \bmod n-k+3}$ as the entry and exit 2-vertices of $A_{t}$, respectively, and properly selecting the entry and exit 2-vertices for all $A_{j}$ 's with $j \neq t$. Q.E.D. 
For ease of description, an $R_{r}=\left[A_{0}, A_{1}, \ldots, A_{n(n-1)(n-2) \cdots(n-k+r+1)-1}\right]$ is said to be good if after a partition each $(r-1)$ vertex of $A_{i}$ is adjacent to one $(r-1)$-vertex of $A_{(i-1) \bmod (n-1)(n-2) \cdots(n-k+r+1)}$ or one $(r-1)$-vertex of $A_{(i+1) \bmod n(n-1)(n-2) \cdots(n-k+r+1)}$.

Lemma 3.4 Suppose $\left|F_{e}\right|=k(n-k)-2$, ard let $S=a_{1} a_{2} \ldots a_{k}$ be a permutation of $1,2, \ldots, k$ so that $\left|D_{a_{1}}\right| \geq\left|D_{a_{2}}\right| \geq \cdots \geq\left|D_{a_{k}}\right|$.If $\left|D_{a_{1}}\right|=\left|D_{a_{2}}\right|=\cdots=$ $\left|D_{a_{k-2}}\right|=n-\bar{k}>5$ and $\left|D_{a_{k-1}}\right|=n-k-1$, there is a fault-free good $R_{1}$ in $A_{n, k}$.

Proof: With the aid of Lemmas $2.4,3.2$ and Lemma 3.3 , the result can be shown.

Q.E.D.

Lemma 3.5 Suppose $\left|F_{e}\right|=k(n-k)-2$, and let $S=a_{1} a_{2} \ldots a_{k}$ be a permutation of $1,2, \ldots, k$ so that $\left|D_{a_{1}}\right| \geq\left|D_{a_{2}}\right| \geq \cdots \geq\left|D_{a_{k}}\right|$. When $k=1$, there is a fault-free hamiltonian cycle for $A_{n, k}$ if $\left|D_{a_{1}}\right|=n-3$. When $k=2$, there is a fault-free hamiltonian cycle for $A_{n, k}$ if $n-k \geq 3$ and $\left|D_{a_{1}}\right|=\left|D_{a_{2}}\right|=n-3$. When $k \geq 3$, there is a fault-free hamiltonian cycle for $A_{n, k}$ if $n-k>5,\left|D_{a_{k-2}}\right|=n-k$, and $\left|D_{a_{k-1}}\right|=\left|D_{a_{k}}\right|=$ $n-k-1$.

Proof: When $k=1, A_{n, 1}$ is a complete graph of $n$ vertices. By Lemma $2.4, A_{n, 1}$ with $\left|D_{a_{1}}\right|=n-3$ faulty edges removed hamiltonian.

When $k=2$, we first apply an $a_{1}$-partition to $A_{n, 2}$ to form a $K_{n}^{1}$. Since $\left|E_{1, a_{1}}\right| \leq\left|D_{a_{1}}\right|=n-3$, the $K_{n}^{1}$ contains at most $n-3$ faulty 1 -edges. By the aid of Lemma 2.4 , a fault-free $R=\left[A_{0}, A_{1}, \ldots, A_{n-1}\right]$, can be determined in $A_{n, 2}$. We use $X=\left\langle x_{1} x_{2} \ldots x_{k}\right\rangle_{n, 1}$, $Y=\left\langle y_{1} y_{2} \ldots y_{k}\right\rangle_{n, 1}$, and $Z=\left\langle z_{1} z_{2} \ldots z_{k}\right\rangle_{n, 1}$ to denote arbitrary three consecutive 1-vertices of the $R_{1}$. Since $x_{\operatorname{dif}(X, Y)} \neq z_{\operatorname{dif}(Y, Z)}$, it is assured by Lemma 3.2 that the $R_{1}$ is good. We then apply an $a_{2}$-partition to the $R_{1}$ so that each $A_{i}(0 \leq i \leq n-1)$ forms a $K_{n-1}^{0}$. Since there are totally $\left|\vec{E}_{0, a_{2}}\right|=\left|D_{a_{2}}\right|=n-3$ faulty edges contained in all $A_{i}$ 's, each $K_{n-1}^{0}$ contains $n-3$ faulty edges at most. Below we construct a fault-free hamiltonian cycle for $A_{n, 2}$ according to three situations.

The first situation is that all $n-3$ faulty edges are located in the same $A_{t}$ for some $0<t<n-1$, and there exists a faulty edge, say $(u, v)$, so that the other $n-4$ faulty edges are incident to $u$ or $v$. We note that $n-2$ vertices of $A_{t}$ are connected to $A_{(t-1) \bmod n}$, and $n-2$ vertices of $A_{t}$ are connected to $A_{(t+1) \operatorname{modn}}$. Moreover, since the $R_{1}$ is good, one vertex of $A_{t}$ is connected to $A_{(t-1) \operatorname{modn}}$ only, another is connected to $A_{(t+1) \operatorname{modn}}$ only, and the others are each connected to both $A_{(t-1) \text { modn }}$ and $A_{(t+1) \text { modn }}$. Thus, it can be assured that one of $u$ and $v$ is connected to $A_{(t-1) \bmod n}$ and the other is connceted to $A_{(t+1) \operatorname{modn}}$, and $v$ is adjacent to a vertex, say $v^{\prime}$, of $A_{(t+1) \operatorname{modn}}$.

We then show that there exists a fault-free hamiltonian path between $u$ and $v$ in $A_{t}$. Since such a path exists for the trivial case of $n=4$, we assume $n \geq 5$.
Let $K^{\prime}$ denote the resulting $K_{n-1}^{0}$ of $A_{t}$ with $n-3$ faulty edges removed, and $H=K^{*}-\{u, v\}$ be a complete graph of $n-3$ vertices. Since $n \geq 5, H$ contains two or more vertices. Let $x$ and $y$ be two vertices of $H$ that are adjacent to $u$ and $v$, respectively. Clearly a hamiltonian path between $x$ and $y$ in $H$ combined with edges $(u, x)$ and $(y, v)$ constitutes a fault-free hamiltonian path between $u$ and $v$ in $A_{t}$.

Since all the other $A_{i}^{\prime} s$ with $i \neq t$ do not contain faulty edges, they are hamiltonian-connected. A faultfree $R_{0}$, i.e., a fault-free hamiltonian cycle, in $A_{n, 2}$ can be generated by taking $u$ and $v$ as the entry and exit vertices of $A_{t}$, respectively, and properly selecting the entry and exit vertices for all $A_{i}$ 's with $i \neq t$.

The second situation is that all $n-3$ faulty edges are located in the $A_{t}$, but the faulty edge $(u, v)$ mentioned above does not exist. By Lemma 2.5, the resulting $K_{n-1}^{0}$ of $A_{t}$ with $n-3$ faulty edges removed contains a fault-free hamiltonian cycle. Let $f$ and $g$ be any two adjacent vertices in the hamiltonian cycle. Since all other $A_{i}$ 's with $i \neq t$ are hamiltonian-connected, a fault-free hamiltonian cycle for $A_{n, 2}$ can be generated by taking $f$ and $g$ as the entry and exit vertices of $A_{t}$, respectively, and properly selecting the entry and exit vertices for all $A_{i}$ 's with $i \neq t$.

The third situation is that $n-3$ faulty edges are distributed over two or more $A_{i}$ 's. By Lemma 2.4, each resulting $K_{n-1}^{0}$ with faulty edges removed is hamiltonian-connected. As described in the proof of Lemma 2.1, a fault-free hamiltonian cycle for $A_{n, 2}$ can be generated by properly selecting the entry and exit vertices for each $A_{i}$.

When $k \geq 3$, we have $\left|D_{a_{1}}\right|=\left|D_{a_{2}}\right|=\cdots=$ $\left|D_{a_{k-3}}\right|=n-k$ because $\left|F_{e}\right|=k(n-k)-2$ and $\left|D_{a_{1}}\right| \geq\left|D_{a_{2}}\right| \geq \cdots \geq\left|D_{a_{k}}\right|$. Since constructing a fault-free hamiltonian cycle for $A_{n, k}$ is very similar to the case of $k=2$, we only show the difference. As before an $R_{1}$ in $A_{r, k}$ is first obtained by executing a $\left(a_{1}, a_{2}, \ldots, a_{k-1}\right)$-partition. Like the case of $k=2$, a good $R_{1}$ is necessary for constructing a fault-free hamiltonian cycle for $A_{n, k}$. But, unlike the case of $k=2$, a good $R_{1}$ is now assured by Lemma 3.4 because $n-k>5$.

Q.E.D.

Similar to the proof of the above, we have the following.

Lemma 3.6 Suppose $\left|F_{e}\right|=k(n-k)-2$, and let $S=a_{1} a_{2} \ldots a_{k}$ be a permutation of $1,2, \ldots, k$ so that $\left|D_{a_{1}}\right| \geq\left|D_{a_{2}}\right| \geq \ldots \geq\left|D_{a_{k}}\right|$. When $k=2$, there is a fault-free hamitionian cycle for $A_{n, k}$ if $\left|D_{a_{1}}\right|=n-k \geq$ 3 and $\left|D_{a_{2}}\right|=n-k-2$. When $k \geq 3$, there is a faultfree hamiltonian cycle for $A_{n, k}$ if $\vec{n}-k \geq 3$ and either $\left|D_{a_{k-1}}\right|=n-k$ and $\left|D_{a_{k}}\right|=n-k-2$ or $\left|D_{a_{k-2}}\right|=$ $n-k,\left|D_{a_{k-1}}\right|=n-k-1$, and $\left|D_{a_{k}}\right|=n-k-2$.

An $R_{r}$ is said to be healthy if after a partition its every $r$-edge contains at least three $(r-1)$-edges that are not faulty.

Lemma 3.7 Suppose $\left|F_{e}\right|=k(n-k)-2$, and let $S=a_{1} a_{2} \ldots a_{k}$ be a permutation of $1,2, \ldots, k$ so that $\left|D_{a_{1}}\right| \geq\left|D_{a_{2}}\right| \geq \cdots \geq\left|D_{a_{k}}\right|$. When $k \geq 3$ and $n \geq$ 
$\frac{3 k+8}{2}$, there is a fault-free hamiltonian cycle for $A_{n, k}$ if $\left|D_{a_{k}}\right| \leq n-k-3$.

Proof: We first prove by induction that healthy rings $R_{k-1}, \quad R_{k-2}, \ldots, R_{1}$ can be generated if a $\left(a_{1}, a_{2}, \ldots, a_{k-1}\right)$-partition is applied to $A_{n, k}$. To begin, an $a_{1}$-partition is applied to $A_{n, k}$, and so a $K_{n}^{k-1}$ results. Since $\left|F_{e}\right|=k(n-k)-2$, we have $\left|D_{a_{1}}\right| \leq k(n-k)-2$. Let $S_{k-1}$ denote the set of those $(k-1)$-edges in $A_{n, k}$ that each contain $n-4$ or more faulty edges. We have $\left|S_{k-1}\right| \leq n-3$ for otherwise $\left|F_{e}\right| \geq(n-4)\left|S_{k-1}\right|>(n-\overline{4})(n-3)>k(n-k)$, which is a contradiction. It is assured by Lemma 2.4 that $K_{n}^{k-1}$ with the set $S_{k-1}$ of $(k-1)$-edges removed is hamiltonian. That is, an $R_{k-1}$ can be obtained whose $(k-1)$-edges each contain at most $n-5$ faulty edges. Since each $(k-1)$-edge of the $R_{k-1}$ comprises $n-2(k-2)$-edges, the $R_{k-1}$ is healthy.

Now we assume a healthy $R_{k-j+1}=$ $\left[A_{0}, A_{1}, \ldots, A_{n(n-1)(n-2) \cdots(n-j+2)-1}\right]$ is obtained after applying a $\left(a_{1}, a_{2}, \ldots, a_{j-1}\right)$-partition to $A_{n, k}$, where $2 \leq j \leq k-1$. Then an $a_{j}$-partition is applied to the $R_{k-j+1}$, and so each $A_{i}(0 \leq i \leq n(n-1)(n-$ 2) $\cdots(n-j+2)-1)$ forms a $K_{n-j+1}^{n-j}$. We note that $\left|D_{a_{j}}\right| \leq\left\lfloor\frac{k(n-k)-2}{j}\right\rfloor$. Let $S_{k-j}$ denote the set of those $(k-j)$-edges in all $A_{i}$ 's that each contain $n-j-3$ or more faulty edges. We have $\left|S_{k-j}\right| \leq n-j-3$ for otherwise $\left|D_{a_{j}}\right| \geq(n-j-3)\left|S_{k-j}\right|>(n-j-3)(n-j-3)>$ $\left\lfloor\frac{k(n-k)-2}{j}\right\rfloor$, which is a contradiction. Then Lemma 2.6 assures that each resulting $K_{n-j+1}^{k-j}$ with the set $S_{k-j}$ of $(k-j)$-edges removed is hamiltonian-connected. Since the $R_{k-j+1}$ is healthy, there are at least three fault-free $(k-j)$-edges between each pair of $A_{i}$ and $A_{(i+1) \operatorname{modn}(n-1)(n-2) \cdots(n-j+2)}$. As a result, we can determine the entry and exit $(k-j)$-vertices, say $X_{i}$ and $Y_{i}$, respectively, for each $A_{i}$ so that $X_{i} \neq Y_{i}$ and the $(k-j)$-edges $\left(Y_{(i-1) \bmod (n-1)(n-2) \cdots(n-j+2)}, X_{i}\right)$ and $\left(Y_{i}, X_{(i+1) \bmod (n-1)(n-2) \cdots(n-j+2)}\right)$ are not faulty. An $R_{k-j}$ can be generated thus for $A_{n, k}$ whose each $(k-j)$-edge contains at most $n-j-4$ faulty edges (and therefore at most $n-j-4$ faulty $(k-j-1)$ edges). Since each $(k-j)$-edge of the $R_{k-j}$ compries $n-j-1(k-j-1)$-edges, the $R_{k-j}$ is healthy.

According to the discussion above, a healthy $R_{1}$ can be obtained after applying a $\left(a_{1}, a_{2}, \ldots, a_{k-1}\right)$-partition to $A_{n, k}$. Then an $a_{k}$-partiton is applied to the $R_{1}$, and its each 1 -vertex forms a $K_{n-k+1}^{0}$. Since there are totally $\left|D_{a_{k}}\right| \leq n-k-3$ faulty edges, Lemma 2.6 assures that each resulting $K_{n-k+1}^{0}$ with faulty edges removed is hamiltonian-connceted. Since $R_{1}$ is healthy, a faultfree $R_{0}$ can be generatedfor $A_{n, k}$ with the same arguments as above.

Q.E.D.

With similar arguments to prove the above lemmas, we have the following results.

Lemma 3.8 Suppose $\left|F_{e}\right|=k(n-k)-2$, and let $S=a_{1} a_{2} \ldots a_{k}$ be a permutation of $1,2, \ldots, k$ so that $\left|D_{a_{1}}\right| \geq\left|D_{a_{2}}\right| \geq \cdots \geq\left|D_{a_{k}}\right|$. When $k \geq 3$, there is a fault-free hamiltonian cycle for $A_{n, k}$ if $n \geq \frac{3 k+8}{2}$ and $\left|D_{a_{k-2}}\right|=\left|D_{a_{k-1}}\right|=\left|D_{a_{k}}\right|=n-k-1$.

Lemma 3.9 Suppose $\left|F_{e}\right|=k(n-k)-2$, and let $S=a_{1} a_{2} \ldots a_{k}$ be a permutation of $1,2, \ldots, k$ so that $\left|D_{a_{1}}\right| \geq\left|D_{a_{2}}\right| \geq \cdots \geq\left|D_{a_{k}}\right|$. When $k \geq 3$, there is a fault-free hamiltonian cycle for $A_{n, k}$ if $n \geq \frac{3 k+8}{2}$ and either $\left|D_{a_{k-1}}\right|=\left|D_{a_{k}}\right|=n-k-2 .\left|D_{a_{k-2}}\right|^{2}=$ $\left|D_{a_{k-1}}\right|=n-k-1$ and $\left|D_{a_{k}}\right|=n-k-2$.

Theorem 3.10 When $k=2$ and $n-k \geq 4$, or $k \geq 3$ and $n \geq \frac{3 k+8}{2}$ (or $n-k \geq 4+\left\lceil\frac{k}{2}\right\rceil$ ), we have $H_{e}\left(A_{n, k}\right)=k(n-k)-2$, which is the best.

Proof: Since the degree of $A_{n, k}$ is $k(n-k)$, $H_{e}\left(A_{n, k}\right) \leq k(n-k)-2$. With the aid of Lemmas $3.5,3.6,3.7,3.8,3.9$, it can be shown that $H_{e}\left(A_{n, k}\right) \geq k(n-k)-2$.

Q.E.D.

The following results can be obtained similarly.

Theorem 3.11 When $k \geq 2$ and $n \geq \frac{3 k+4}{2}$ (or $n-$ $\left.k \geq 2+\left\lceil\frac{k}{2}\right\rceil\right)$, we have $H_{e}\left(A_{n, k}\right)=k(n-k-3)-1$.

Theorem 3.12 When $k \geq 2$ and $n-k \geq 3$, we have $H_{e}\left(A_{n, k}\right) \geq k$.

\section{References}

[1] S. B. Akers, D. Harel and B. Krishnamurthy, "The star graph: an attractive alternative to the $n$ cube," Proceedings of the International Conference on Parallel Processing, 1987, pp. 393-400.

[2] F. Buckley and F. Harary, "Distance in Graphs," Addison-Wesley, 1989.

[3] K. Day and A. Tripathi, "Characterization of node disjoint paths in arrangement graphs," Technical Report TR 91-43, Computer Science Department, University of Minnesota, 1991.

[4] K. Day and A. Tripathi, "Arrangement graphs: a class of generalized star graphs," Information Processing Letters, vol. 42, no. 5, pp. 235-241, 1992.

[5] K. Day and A. Tripathi, "Embedding of cycles in arrangement graphs," IEEE Transactions on Computers, vol. 12, no. 8, pp. 1002-1006, 1992.

[6] K. Day and A. Tripathi, "Embedding grids, hypercubes, and trees in arrangement graphs," Proceedings of the International Conference on Parallel Processing, 1993, pp. III-65-III-72.

[7] R. Fernandes, D. K. Friesen, and A. Kanevsky, "Embedding rings in recursive networks," Proceedings of the International Conference on Parallel Processing, 1994, pp. I-51-I-58.

[8] S. Latifi, S. Q. Zheng, and N. Bagherzadeh, "Optimal ring embedding in hypercubes with faulty links," Proceedings of the IEEE Symposium on Fault-Tolerant Computing, 1992, pp. 178-184. 
[9] R. A. Rowley and B. Bose, "Fault-tolerant ring embedding in deBruijn networks," IEEE Transcations on Computers, vol. 12, no. 2, pp. 1480-1486, 1993.

[10] Y. C. Tseng, S. H. Chang, and J. P. Sheu, "Faulttolerant ring embedding in star graphs," Proceedings of the International Parallel Processing Symposium, 1996, pp. 660-665.

[11] M. Paoli, W. W. Wong, and C. K. Wong, "Minimum $k$-hamiltonian graphs II," Journal of Graph Theory, vol. 10, no. 1, pp. 79-95, 1986. 